\title{
Bilinear reduced order approximate model of parabolic distributed solar collectors
}

\author{
S. Elmetennani ${ }^{\mathrm{a}}$, T.M. Laleg-Kirati ${ }^{\mathrm{a}}$
}

${ }^{a}$ Computer, Electrical and Mathematical Sciences and Engineering Division, King Abdullah University of Science and Technology, 4700 Thuwal, Jeddah, Saudi Arabia

\begin{abstract}
This paper proposes a novel, low dimensional and accurate approximate model for the distributed parabolic solar collector, by means of a modified gaussian interpolation along the spatial domain. The proposed reduced model, taking the form of a low dimensional bilinear state representation, enables the reproduction of the heat transfer dynamics along the collector tube for system analysis. Moreover, presented as a reduced order bilinear state space model, the well established control theory for this class of systems can be applied. The approximation efficiency has been proven by several simulation tests, which have been performed considering parameters of the Acurex field with real external working conditions. Model accuracy has been evaluated by comparison to the analytical solution of the hyperbolic distributed model and its semi discretized approximation highlighting the benefits of using the proposed numerical scheme. Furthermore, model sensitivity to the different parameters of the gaussian interpolation has been studied.
\end{abstract}

Keywords: Solar collector, Solar energy, Model reduction, Bilinear model, Hyperbolic partial differential equation

\section{Introduction}

The increasing interest in solar energy is driven by the need of renewable clean sources in order to meet the exponential growth of energy demand. Indeed, solar energy is a promising alternative able to reduce the reliance on fossil fuels and the environmental damages due to carbon emissions. Several plants are already successfully operating or under construction, where most of them are based on the parabolic distributed technology. A parabolic trough is a collector, which uses parabolic shaped mirrors to concentrate the received solar power on a receiver tube placed in the focal line of the parabola. The concentrated solar irradiance is then absorbed by a thermal carrier fluid flowing along the collector to transport the generated energy to the storage system or to feed a thermal process.

Modeling of concentrated distributed collectors has already been subject of numerous publications. Indeed, several models of the parabolic solar collector exist in the literature, with different levels of accuracy and complexity [1, 2, 3, 4]. The existing models vary from the simplest ones, based on linear steadystate relationships to nonlinear distributed ones. One particular representation, which has been widely used, is a first order hyperbolic partial differential equation (PDE) describing the heat transfer along the collector where the fluid temperature is considered as the state variable. This mathematical representation describing the system dynamics has been obtained from an energy balance analysis neglecting the diffusion phenomenon

\footnotetext{
Research reported in this publication has been supported by King Abdullah University of Science and Technology (KAUST).

Email addresses: shahrazed.elmetennani@kaust.edu.sa (S. Elmetennani), taousmeriem.laleg@kaust.edu.sa (T.M. Laleg-Kirati)
}

with respect to the heat transport dynamics and the heat loss with the external environment. However, the distributed nature of the system subject to random external working conditions makes the model difficult to analyze and not convenient for control design. Thereafter, the solution commonly adopted is approximation of the distributed parameters equation by numerical schemes, generally leading to high dimensional state vectors.

In fact, apart from the classical semi discretization numerical scheme [5, 6, 7], numerous approximation techniques have been used to reduce the dimension of the distributed model of the solar collector. Indeed, the hyperbolic PDE has been reduced to a lumped parameter model by Igreja et al.[8] who applied orthogonal collocation on the PDE representing the plant dynamics. Moreover, Silva et al.[9] adopted a time scaling discretization scheme to obtain a reduced state space representation. The approach followed in this study is new with respect to the numerical schemes that have already been used for model reduction of the distributed collector. The main objective in introducing the present new technique is to compromise between the accuracy of the dynamics approximation and the simplicity of the resulting representation in order to make the control design easier but yet efficient. Indeed, this new approximation method provides a low dimensional state space model, to describe the heat transfer dynamics in the solar collector, with reduced information loss and less computational complexity making the method very competitive to the existing approximation approaches. The proposed numerical scheme allows model reduction of the hyperbolic PDE to a low dimensional bilinear state space representation. Explicit use is made of the hyperbolic distributed model to derive a low dimensional set of ODEs by application of a modified version of the gaussian interpola- 
tion. A gaussian radial basis is defined to express the correlation between the coordinates in the spatial domain. Then, in order to reproduce the time varying system dynamics, a least square minimization problem is formulated leading to a state representation describing the time evolution of the interpolation weighting parameters and consequently defining the heat transfer mechanism.

From a more general perspective, the proposed lumped model could be used to simplify the control design not only for the solar collector but for a larger category of hyperbolic conductive systems in which transport phenomena of mass and energy occurs in one direction with no diffusion. For instance, moisture control, glass-tube manufacture, aerosol processes, gas absorbers, traffic-highway flows.

This paper is organized as follows. First, the solar plant is described in section 2 . Then, the distributed model and its semi discrete approximate model are presented in section 3. Subsequently, the reduced order bilinear approximate model is derived in section 4 using the dynamical gaussian interpolation followed by numerical tests to evaluate its accuracy and its performance in section 5. Finally, section 6 summarizes the obtained results.

\section{Plant description}

A solar plant, based on parabolic troughs technology, collects solar energy by exploiting the thermal properties of the fluid flowing through the parabolic distributed collectors. The solar collector is composed of parabolic reflective surfaces concentrating the solar irradiation onto a receiver tube positioned in the focal line of the parabola. The collector tube consists

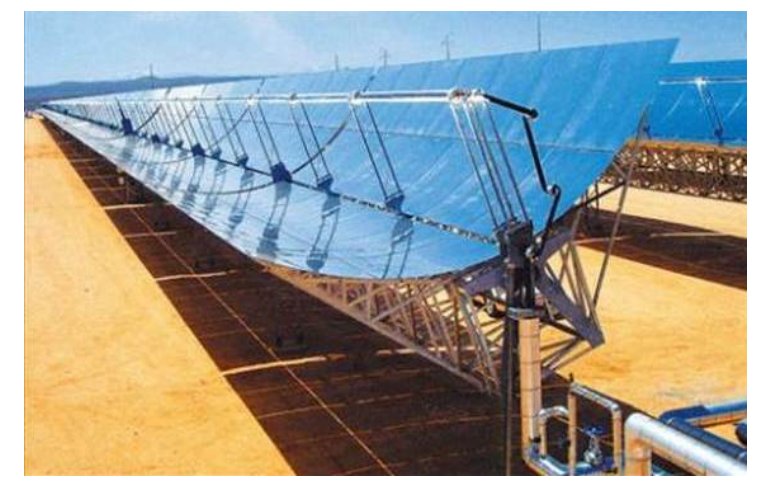

Figure 1: Parabolic distributed solar collector

of a pipe wrapped with a selective coating to enhance the heat absorption, which is covered by a glass envelop that maintains the vacuum in the annulus in order to reduce the energy losses. Fig. 1 displays a functioning loop using a parabolic distributed collector.

A hydraulic circuit constituting the energy production loop generally connects several lines of distributed collectors in parallel, which usually have similar characteristics, receiving the thermal fluid from a distribution, known as the cold header pipe. The fluid is then routed through the different collectors to be heated and returned at higher temperatures to another pipe, known as the hot header pipe, which feeds a thermal process or is connected to the storage system. Fig. 2 depicts a schematic of a hydraulic loop with a concentrated distributed solar collector.

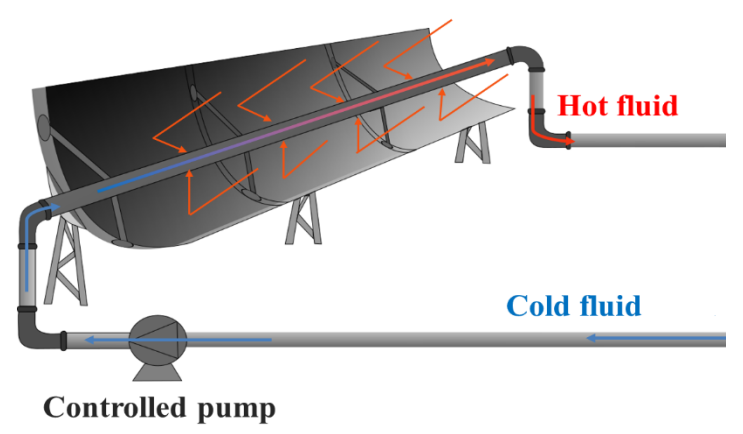

Figure 2: Solar thermal hydraulic circuit

\section{Plant modeling}

\subsection{Distributed model}

A first order hyperbolic transport equation is used to describe the heat transfer phenomenon in the parabolic collector. Based on an energy balance analysis, the dynamics of the process, which are represented by the evolution of the heat transport fluid temperature, are given by [3, 2]:

$$
\left\{\begin{array}{l}
T_{t}(x, t)+\frac{Q(t)}{A_{s}} T_{x}(x, t)=\frac{v_{0} G}{\rho c A_{s}} I(t), \quad x \in[0, L], \quad t \in \mathbb{R}^{+} \\
T(0, t)=T_{\text {in }}(t) \\
T(L, t)=T_{\text {out }}(t)
\end{array}\right.
$$

where

$\begin{array}{ll}t & \text { Time }(s) \\ x & \text { Space }(m) \\ T(x, t) & \text { Fluid temperature }\left({ }^{\circ} \mathrm{C}\right) \\ I(t) & \text { Solar irradiance }\left(\mathrm{W} / \mathrm{m}^{2}\right) \\ Q(t) & \text { Volumetric fluid flow rate }\left(\mathrm{m}^{3} / s\right) \\ \rho & \text { Density }\left(\mathrm{kg} \mathrm{m} \mathrm{m}^{-3}\right) \\ c & \text { Specific heat capacity }\left(\mathrm{J} \mathrm{C} \mathrm{C}^{-1} \mathrm{~kg}^{-1}\right) \\ A_{s} & \text { Cross-sectional area }\left(\mathrm{m}^{2}\right) \\ v_{0} & \text { Mirrors optical efficiency } \\ G & \text { Mirrors optical aperture }(m) \\ L & \text { Length of the collector tube }(m) \\ T_{\text {in }}(t) & \text { Inlet fluid temperature }\left({ }^{\circ} \mathrm{C}\right) \\ T_{\text {out }}(t) & \text { Outlet fluid temperature }\left({ }^{\circ} \mathrm{C}\right)\end{array}$

also, $T_{t}(x, t) \equiv \frac{\partial T(x, t)}{\partial t}$ and $T_{x}(x, t) \equiv \frac{\partial T(x, t)}{\partial x}$ denote the first derivatives with respect to time and space respectively. $s(t)=\frac{v_{0} G}{\rho c A_{s}} I(t)$ is the source term affecting the heat transport dynamics, which depends on the solar irradiance $I(t) . u(t)=\frac{Q(t)}{A_{s}}$ is the fluid velocity accessible for tuning by changing the fluid volumetric flow rate $Q(t)$.

Integrating the solution of equation (1) along the characteristic curves, such that $(x, t)$ lies in the upper half-plane $\mathbb{R}^{2+}:=$ 


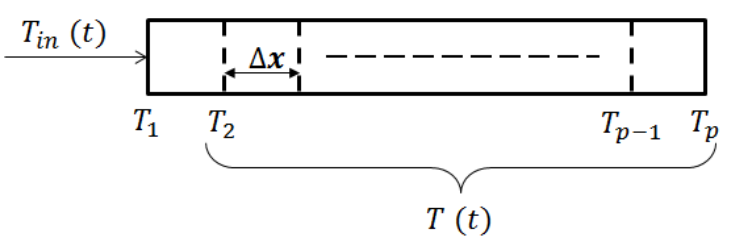

Figure 3: Spatial discretization subintervals

$(-\infty, \infty) \times[0, \infty), T: \mathbb{R}^{2+} \rightarrow \mathbb{R}$ and $u: \mathbb{R}^{+} \rightarrow \mathbb{R}$ is a continuous function sufficiently smooth, the analytical solution describing the temperature profile is given by [9]:

$$
\left\{\begin{array}{l}
\text { if } x-\int_{0}^{t} u(\tau) d \tau \geq 0: \\
T(x, t)=T\left(x-\int_{0}^{t} u(\tau) d \tau, 0\right)+\int_{0}^{t} s(\tau) d \tau, \\
\text { else if } x-\int_{0}^{t} u(\tau) d \tau<0: \\
T(x, t)=T\left(0, t^{*}\right)+\int_{t^{*}}^{t} s(\tau) d \tau, \text { where } \int_{t^{*}}^{t} u(\tau) d \tau=x .
\end{array}\right.
$$

\subsection{Eulerian approximate state representation}

Considering the model (1), the fluid temperature describing the thermal dynamics in the system can be reproduced using an Eulerian state space model by partitioning the receiver to a finite number of similar intervals of dimension $\Delta x$ defined by a one dimensional grid of $p$ equidistant points (see Fig.3). Thus, using the backward Euler spatial discretization of the domain $[0, L]$, the finite difference approximation yields to the nonlinear state-space model of dimension $(p-1)$ :

$$
\dot{T}=\left(A_{d} T+E\right) u(t)+B_{d}(t), \quad y(t)=T_{p}(t),
$$

where $T(t)=\left[T_{2}(t), \ldots, T_{p}(t)\right]$ is the vector of the state variable,

$$
\begin{gathered}
A_{d}=-\frac{1}{\Delta x}\left[\begin{array}{ccccc}
1 & 0 & 0 & \ldots & 0 \\
-1 & 1 & 0 & \ddots & \vdots \\
0 & -1 & \ddots & \ddots & \vdots \\
\vdots & \ddots & \ddots & \ddots & \vdots \\
0 & \ldots & 0 & -1 & 1
\end{array}\right], \\
E=\left[\begin{array}{llll}
-\frac{T_{i n}}{\Delta x} & 0 & \ldots & 0
\end{array}\right]^{\top}, \quad B_{d}(t)=\frac{v_{0} G}{\rho c A_{s}} I(t) \mathbf{1}_{p \times 1},
\end{gathered}
$$

with $T_{i}(t)=T\left(x_{i}, t\right)$ representing the temperature approximation at the different positions $x_{i}$ along the receiver $x_{i}=(i-1) \Delta x$ for $i=\{2, \ldots, p\}$ and $\Delta x=\frac{L}{p-1} \cdot \mathbf{1}_{p \times 1}$ denote the unit vector of dimension $(p \times 1)$.

For control design, the reproduction of the transient behavior of the system, especially under varying working conditions, is important to ensure the efficiency of the controller. Therefore, in order to achieve the desired accuracy, a high number of knots might be required leading to high dimensional state vectors, which increases the computational cost and control design complexity.

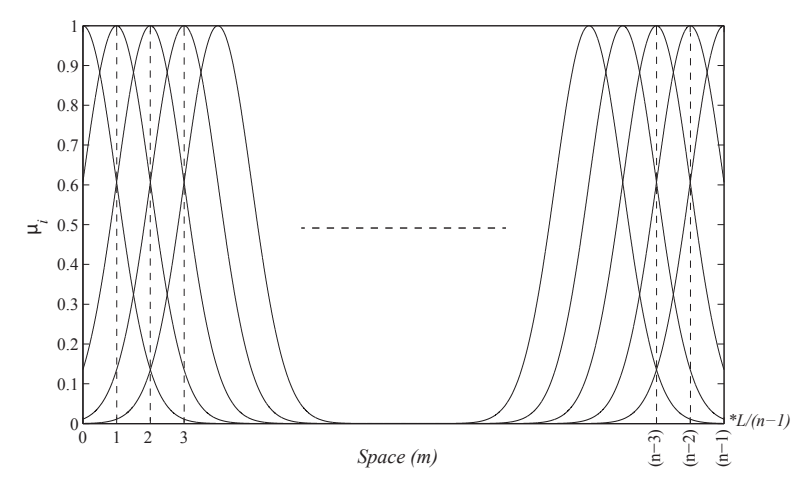

Figure 4: Distribution of the gaussian functions along the collector tube

\section{Reduced bilinear approximate model}

This section presents the main idea and steps in developing a bilinear reduced model of the solar collector.

First, define $n$ sets $D_{i}$ in the space $\Omega$ characterized by distribution functions $\mu_{i}$ defined in terms of the spatial coordinates, such that:

$$
D_{i}=\left\{\left(x, \mu_{i}(x)\right) \mid x \in[0, L]\right\}, \quad i=\{1, \ldots, n\} .
$$

These functions have been chosen to be gaussian in order to conserve the characteristics of the distributed solution; smoothness, continuity and infinite differentiability. They are expressed in terms of the spatial positions as follows:

$$
\mu_{i}(x)=\exp \left(\frac{1}{2}\left(\frac{x-a_{i}}{\sigma_{i}}\right)^{2}\right), \quad i=\{1, \ldots, n\}
$$

where $a_{i}$ and $\sigma_{i}$ are the mean value and the variance of the gaussian function corresponding to the $i^{\text {th }}$ set.

In this work, for $i=\{1, \ldots, n\}$, the mean value and the variance are defined by:

$$
a_{i}=(i-1) \frac{L}{(n-1)}, \quad \text { and } \quad \sigma_{i}=\sigma=\alpha \frac{L}{(n-1)},
$$

$\alpha$ is a scaling parameter.

It is worth to mention that the gaussian functions are centered around equidistant points along the spatial domain with equal variance values covering half of the interval between two consecutive gaussian centers. Fig. 4 illustrates the distribution of the radial functions along the collector with the previous configuration.

Thereafter, in order to reproduce the temperature dynamics, the overall approximate output at each position $x$ along the collector is interpolated using the radial basis functions defining the sets $D_{i}$ for $i=\{1, \ldots, n\}$. Thus, the approximate value of the temperature $\hat{T}(x, t)$ is given by:

$$
\begin{aligned}
\hat{T}(x, t) & =\sum_{i=1}^{n} \xi_{i}(t) \gamma_{i}(x), \\
& =\gamma^{\top}(x) \xi(t),
\end{aligned}
$$


with $\gamma(x)=\left[\gamma_{1}(x), \ldots, \gamma_{n}(x)\right]$ and $\xi(t)=\left[\xi_{1}(t), \ldots, \xi_{n}(t)\right]$, where

$$
\gamma_{i}(x)=\frac{\mu_{i}(x)}{\sum_{k=1}^{n} \mu_{k}(x)} .
$$

Substituting the approximate solution $\hat{T}(x, t)$ in the physical distributed model (1) yields to:

$$
\begin{aligned}
\frac{\partial}{\partial t} \hat{T}(x)+u(t) \frac{\partial}{\partial x} \hat{T}(x, t) & =s(t), \\
\gamma(x)^{\top} \dot{\xi}(t)+u(t) \xi^{\top}(t) \gamma_{x}(x) & =s(t),
\end{aligned}
$$

and

$$
\hat{T}(0, t)=\gamma^{\top}(0) \xi(t)=T_{\text {in }}(t) .
$$

Thus, considering a one dimensional grid of $p$ equidistant points on the domain $[0, L], j=\{0, \Delta x, 2 \Delta x, \ldots,(p-1) \Delta x, L\}$ with $\Delta x=\frac{L}{p-1}$, one obtains:

$$
\Gamma \dot{\xi}+\Gamma_{x} \xi u(t)=S(t)
$$

such that:

$$
\begin{gathered}
\Gamma=\left[\begin{array}{ccc}
\gamma_{1}(0) & \ldots & \gamma_{n}(0) \\
\gamma_{1}(\Delta x) & \ldots & \gamma_{n}(\Delta x) \\
\vdots & & \vdots \\
\gamma_{1}(j) & \ldots & \gamma_{n}(j) \\
\vdots & & \vdots \\
\gamma_{1}(L) & \ldots & \gamma_{n}(L)
\end{array}\right]_{p \times n} \\
\Gamma_{x}=\left[\begin{array}{ccc}
\gamma_{x 1}(0) & \ldots & \gamma_{x n}(0) \\
\gamma_{x 1}(\Delta x) & \ldots & \gamma_{x n}(\Delta x) \\
\vdots & & \vdots \\
\gamma_{x 1}(j) & \ldots & \gamma_{x n}(j) \\
\vdots & & \vdots \\
\gamma_{x 1}(L) & \ldots & \gamma_{x n}(L) \\
S(t)= & s(t) \mathbf{1}_{p \times 1}
\end{array}\right]_{p \times n}
\end{gathered}
$$

and

$$
\gamma_{x i}(x)=\frac{\partial \gamma_{i}(x)}{\partial x} .
$$

Subsequently, in order to ensure that the proposed approximation is accurately reproducing the distributed behavior of the temperature, the values of the parameters $\xi(t)$ are chosen such that to minimize the approximation error of system (10) in a least squares sense. Therefore, the time evolution of the parameters $\xi(t)$ is given by the following bilinear state space representation, which describes the dynamics of the parabolic solar collector, such that:

$$
\left\{\begin{array}{c}
\dot{\xi}(t)=A \xi(t) u(t)+B(t) \\
y(t)=C \xi(t)
\end{array}\right.
$$

where

$$
A=-\left(\Gamma^{\top} \Gamma\right)^{-1} \Gamma^{\top} \Gamma_{x}, \text { and } B(t)=\left(\Gamma^{\top} \Gamma\right)^{-1} \Gamma^{\top} S(t) .
$$

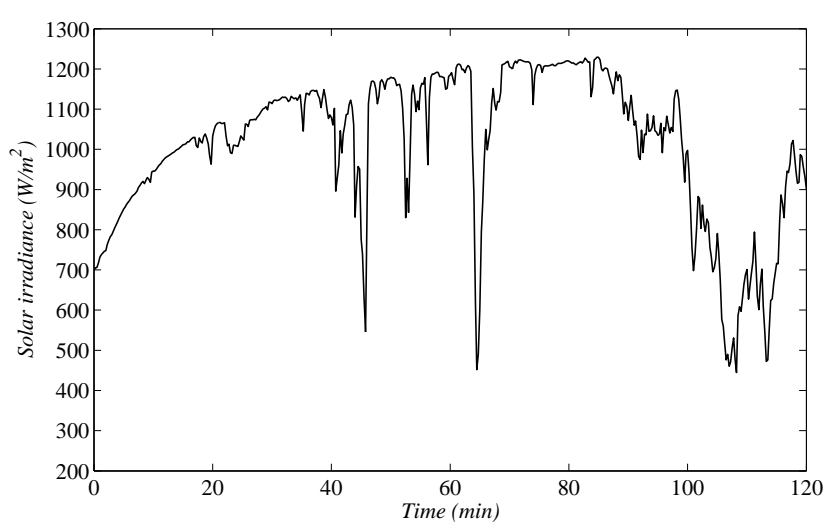

Figure 5: Profile of the solar irradiance

The system output representing the outlet fluid temperature is given by:

$$
y(t)=C \xi(t)=T(L, t) \text { with } C=\left[\gamma_{1}(L) \ldots \gamma_{m}(L)\right] .
$$

It is worth to point out that the system order is equal to the number of input sets $n<p$, which is generally less than ten (10). Moreover, this state dimension is relatively small comparing to other numerical schemes, particularly the semi discretization which usually requires a high state dimension in order to ensure the accuracy of the approximation.

The numerical approach followed, in this study, uses a dynamical gaussian interpolation for the PDE model reduction. Considering the fact that gaussian functions are radial basis distributions, this approach can be classified under Kansa's technique for PDEs approximation [10], where radial basis interpolation is used to numerically approximate PDEs solutions.

\section{Numerical results}

In order to perform the numerical simulations, we have considered the parameters of the Acurex Field Del Almeria. The later, considered as a typical solar field based on parabolic troughs technology, is a solar power field situated in Tabernas in Spain, mainly used for research purposes. For more details the reader can refer to [2].

Several tests have been carried out along 120 minutes to evaluate the accuracy and the sensitivity of the proposed reduced bilinear model. The system has been simulated under varying solar irradiance profile characterized by smooth variations with some sudden unfavorable changes (see Fig.5). Moreover, the bilinear model describing the heat transport has been tested for different velocities by changing the flow rate of the fluid circulating throughout the collector. In that respect, the flow rate has been considered varying in time. The experiment starts with an average flow rate of $7 \mathrm{~m}^{3} / \mathrm{s}$, which is reduced later to $5 \mathrm{~m}^{3} / \mathrm{s}$ and increased back until it reaches $10 \mathrm{~m}^{3} / \mathrm{s}$ (see Fig.6).

It should be noted that all numerical tests in this study have been run using the irradiance profile of Fig.5 and the flow rate changes shown in Fig.6. Besides, the sets of ODEs generated by the approximation schemes are numerically implemented using euler finite differences with a time step of (0.01) second. 


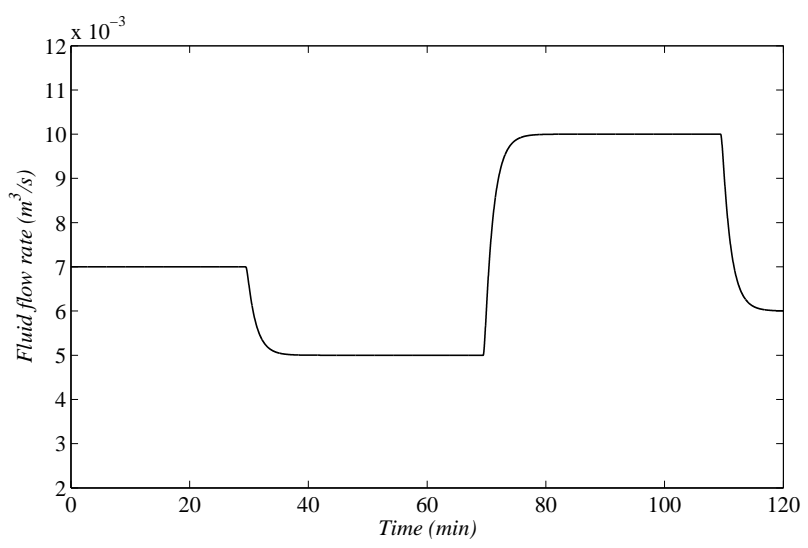

Figure 6: Profile of the fluid flow rate

\subsection{Test 1: Model validation}

The first test has been performed in order to validate the proposed reduced model. For this purpose, the temperature evolution has been calculated at different positions along the collector $\left(\frac{L}{5}, \frac{2 L}{5}, \frac{3 L}{5}, \frac{4 L}{5}, L\right)$ and then compared to the analytical solution of the hyperbolic heat transport equation. Moreover, the performance evaluation of the bilinear approximate model reproducing the heat transfer dynamics has been investigated. Therefore, the approximation error has been analyzed by comparison to the numerical error induced by the semi discretization scheme. A grid of 500 knots has been considered for the computations of both numerical schemes, the gaussian interpolation based scheme and the eulerian semi discretization. Then, the bilinear model has been generated with a set of 8 gaussian functions resulting in a state vector of dimension $(n=8)$. It will be shown later that increasing the number of sets requires more computational effort without significant improvement in the performance. The variance has been chosen equal to $\sigma=\frac{L}{n-1}$. A detailed analysis will be performed to address the effect of the variance on the accuracy of the approximation. The temperature profiles generated by this test are represented in Fig.7a and Fig. $7 b$ where the temperature evolution is depicted for 5 different points. The responses seem be to identical despite the variations in the working conditions and the external disturbances. However, the general dynamics are well reproduced with both approximation techniques. For this purpose, a graphical analysis of the approximation error for both numerical schemes has been performed, (see Fig.8), showing that the performance of the bilinear model is equivalent to the one presented by the eulerian state representation. Furthermore, the same accuracy level could be achieved with a state dimension equal to 8 comparing to a state vector of 500 components.

The control objective generally addressed for parabolic distributed solar collector aims at forcing the outlet temperature to track a set reference. Thereafter, reproducing accurately the time evolution of the outlet temperature is of great interest for control design. The profile of the approximation error generated by both schemes at the outlet of the tube can be appreciated by looking at the histograms in Fig.9 illustrating that the density of both errors is very high in the neighborhood of zero

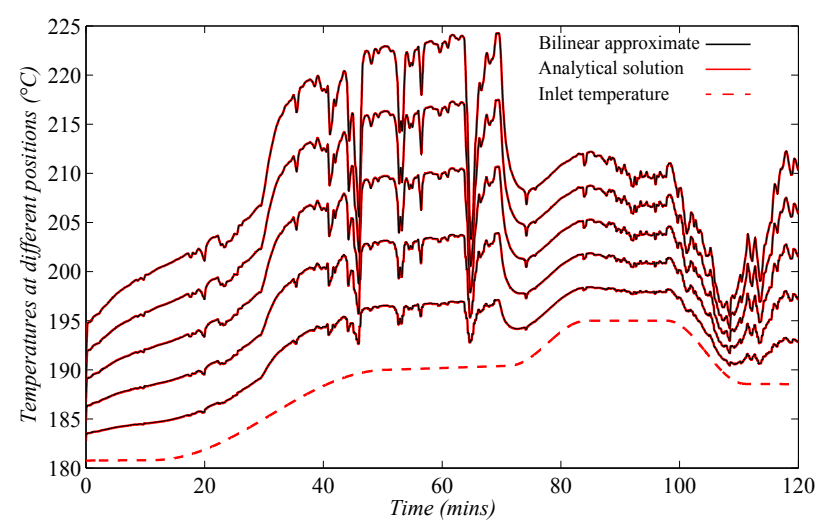

(a) Bilinear approximate model

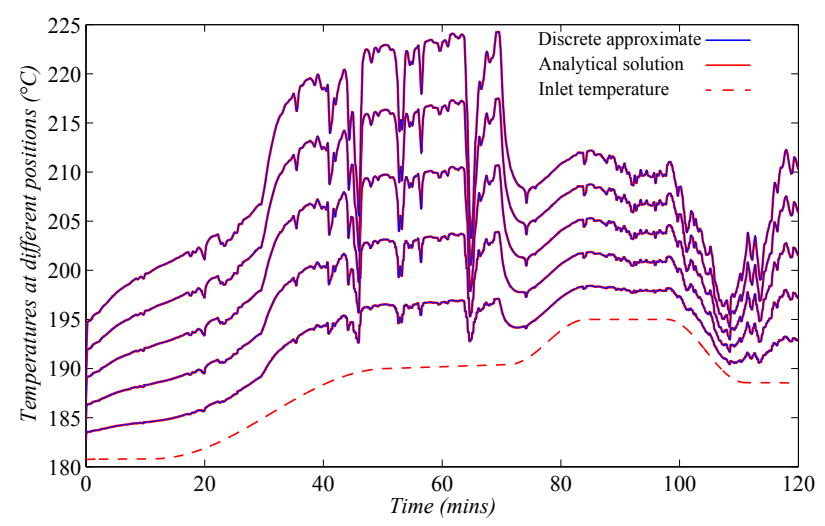

(b) Semi discrete solution

Figure 7: Validation test

bounded by $( \pm 0.01)$. The semi discrete model presents relatively a better performance comparing to the proposed bilinear model, presenting a smaller density of the error in the interval ([-0.02, $-0.01[\cup] 0.01,0.02])$. Alternatively, the computations of the bilinear model has been run with a state vector of dimension (8) to approximate the temperature of (500) points while the semi discrete approximate solution, for the same grid, has used a state vector of dimension (500). Indeed, the time required for data generation using a standard desktop computer $(2.70 \mathrm{GHz})$ for a 120 minutes simulation test was estimated to 43 seconds for the bilinear model and 66 seconds for the semi discrete approximation. Therefore, the accuracy of the gaussian interpolation scheme is acceptable and the reduction of the computational effort compromises the information loss.

Summarizing the results obtained in the first test, the proposed reduced model derived from the hyperbolic transport equation presents very good correlation with its analytical solution despite the varying working conditions. The results show that the accuracy of the error is acceptable but it is interesting to investigate if the approximation can be improved by changing the parameters of the gaussian radial basis functions. Thereafter, in order to study the sensitivity of the proposed bilinear model with respect to different parameters, the system has been tested for different configurations of the radial basis scheme. 

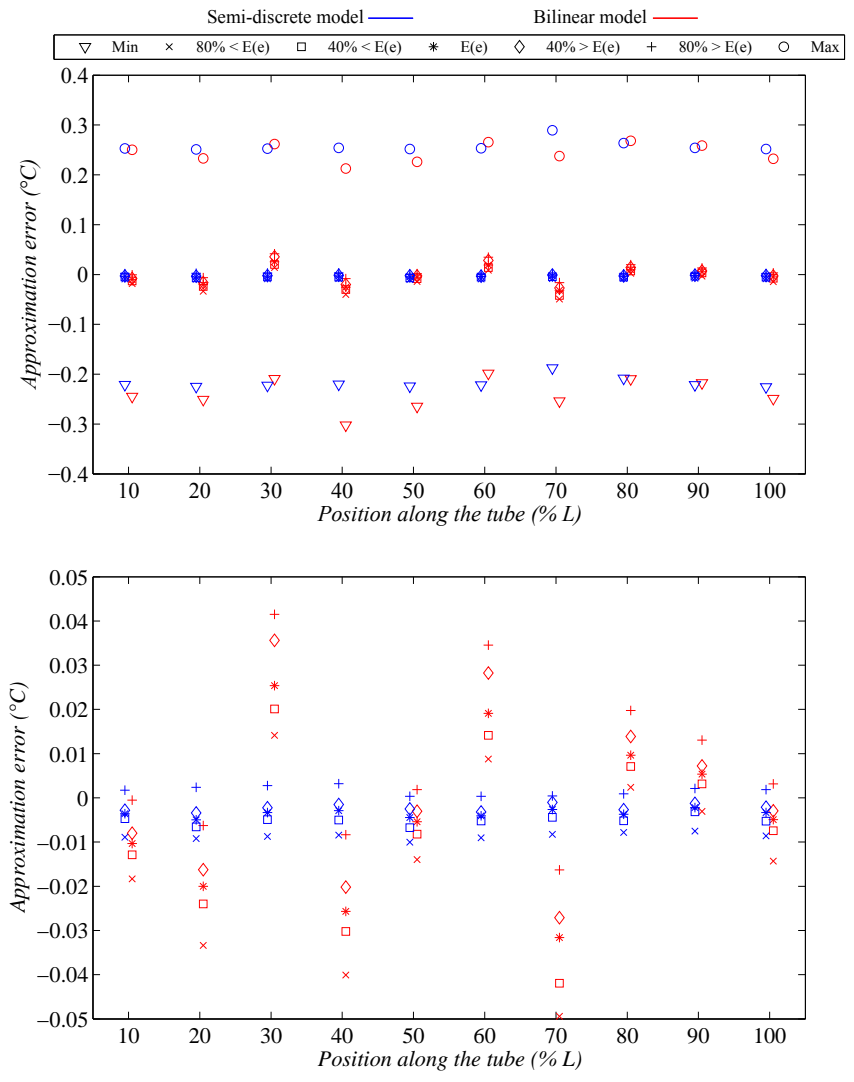

Figure 8: Graphical analysis of the approximation error

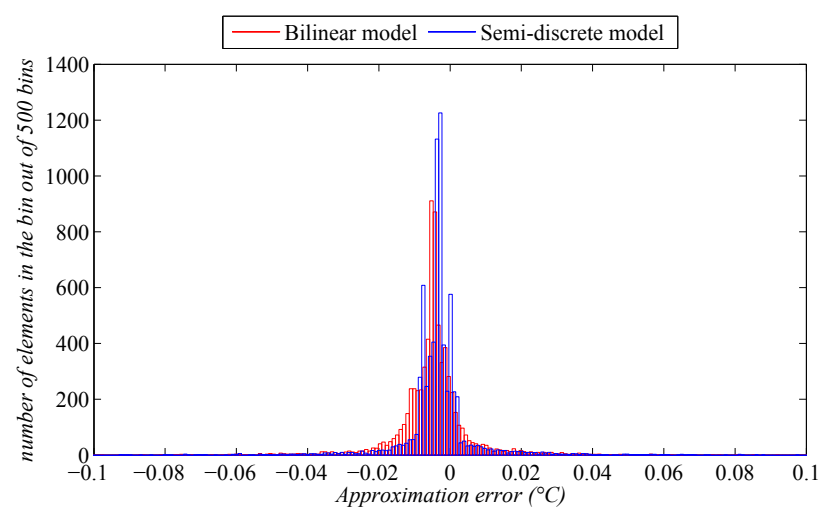

Figure 9: Histogram of the approximation error at the tube outlet

\subsection{Test 2: Effect of the number of sets}

The second test carried out aims at studying the effect of the number of sets on the accuracy of the approximation. Thus, the temperature profile has been reproduced by the bilinear model at different positions along the collector and then compared to the analytical solution describing the thermal dynamics. Considering the same parameters as the previous test $(p=500$ and $\sigma=\frac{L}{n-1}$ ), the simulations have been repeated for different numbers of sets. The obtained results are illustrated in Fig.10a, which shows that the mean value of the error for all points is enveloped by a decaying function converging to zero as $n$ goes

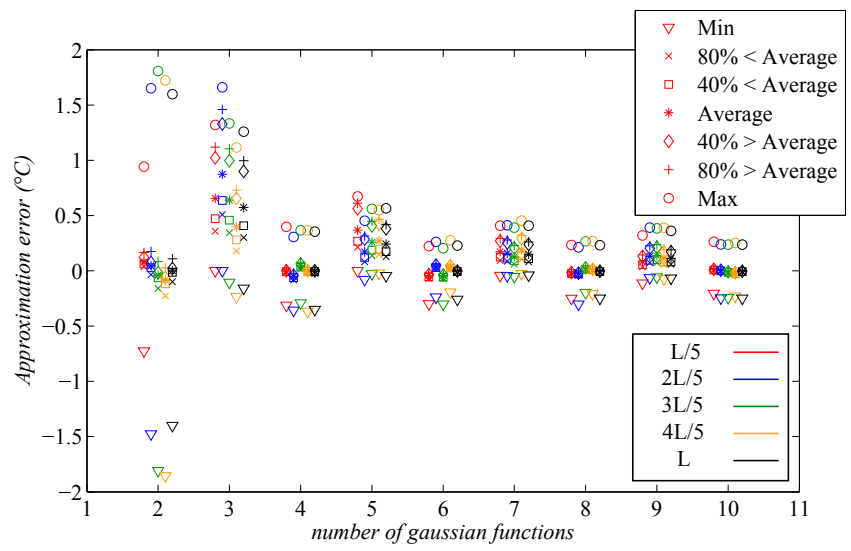

(a) Approximation error of Test2

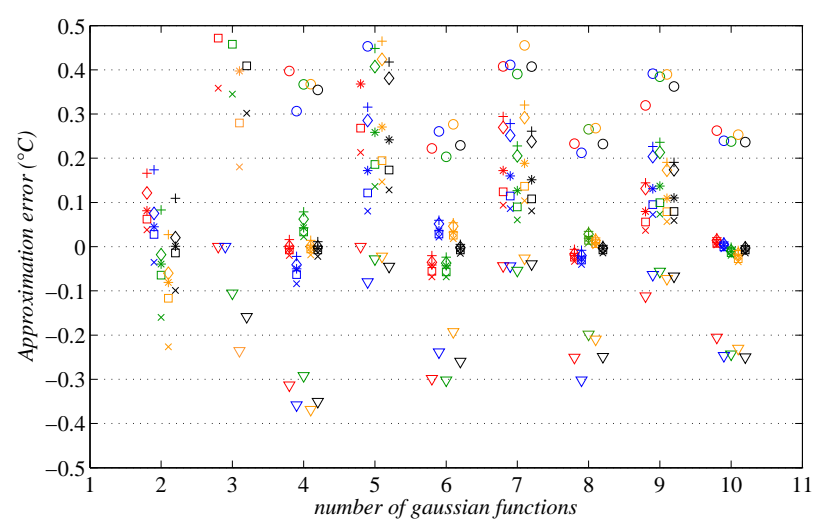

(b) Zoom on the results of Test2

Figure 10: Effect of the number of gaussian functions on the approximation error

bigger. Moreover, Fig.10b shows that (8) radial basis functions would be enough to reproduce the heat transfer with satisfactory accuracy while keeping the computational effort as low as possible. Indeed, increasing the number will be computationally more expensive while the improvement in the approximation accuracy is insignificant.

\subsection{Test 3: Effect of the variance of the gaussian functions}

Now, we study the effect of the variance on the approximation accuracy level. Therefore, to assess the impact of the variance, the temperature has been approximated at different positions for several configurations. The grid size (500) and the number of radial functions (8) have been kept unchanged, only the variance of the gaussian functions has been modified, ranging from $\left(0.4 \frac{L}{n-1}\right)$ to $\left(1.5 \frac{L}{n-1}\right)$. The obtained data series are presented in Fig.11 where each figure corresponds to a particular position on the tube and displays the time evolution of the temperature for different values of the variance .

The figures show that the approximation error is increasingly converging to zero as the variance of the gaussian function is getting bigger. Moreover, acceptable accuracy can be clearly noticed starting a variance value equal to $\left(\frac{L}{n-1}\right)$ with better performance as the variances increases. Thereafter, it can be con- 


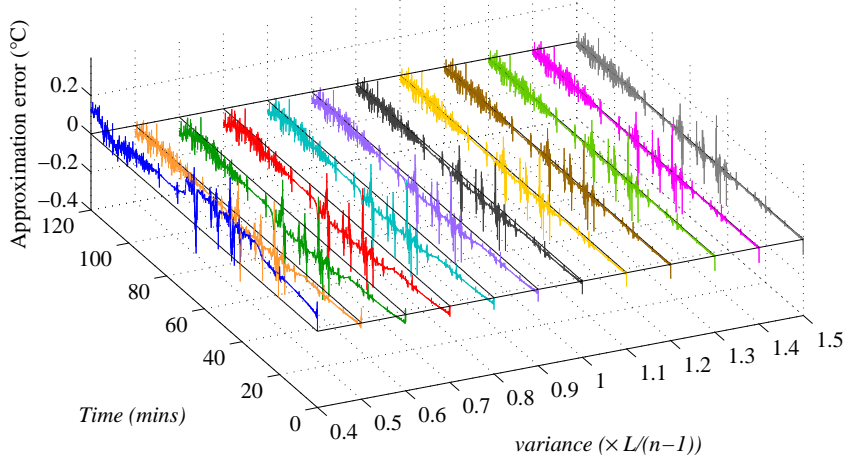

(a) $\mathrm{x}=\mathrm{L} / 5$

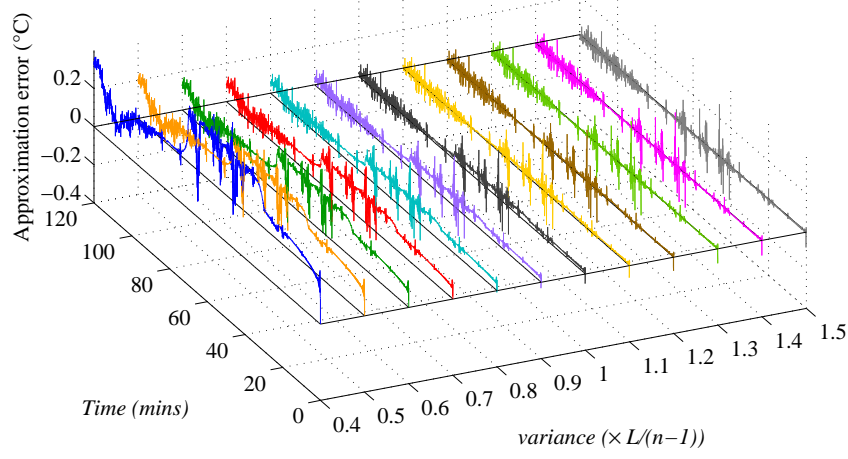

(c) $x=3 L / 5$

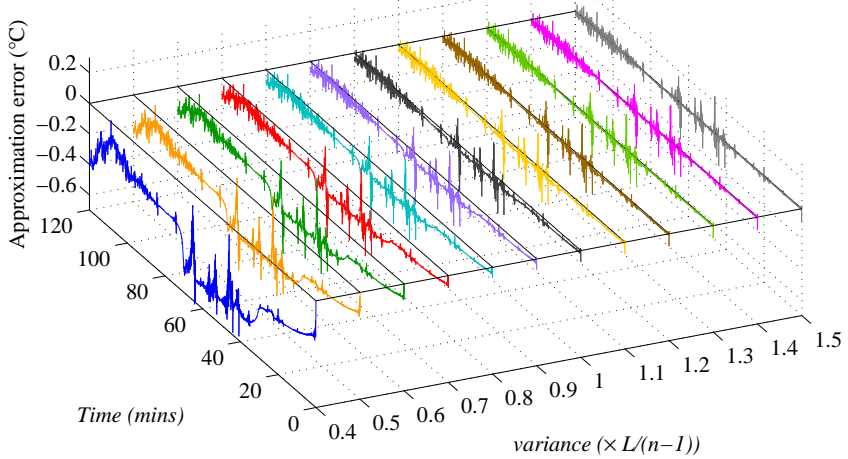

(b) $\mathrm{x}=2 \mathrm{~L} / 5$

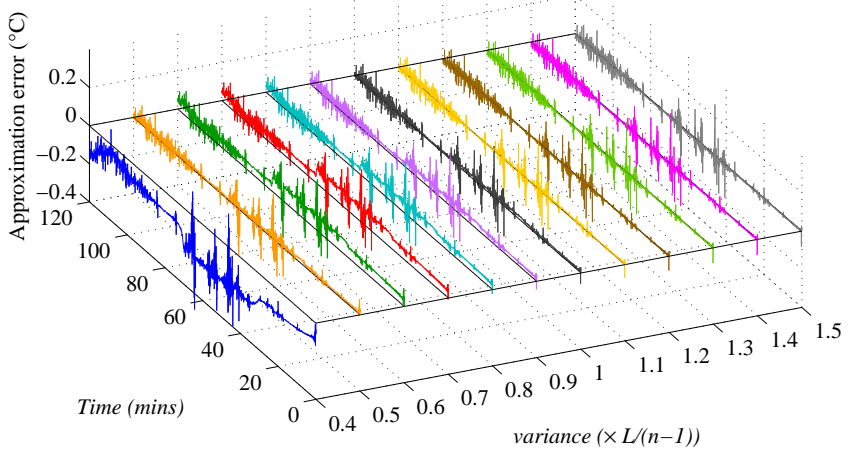

(d) $\mathrm{x}=4 \mathrm{~L} / 5$

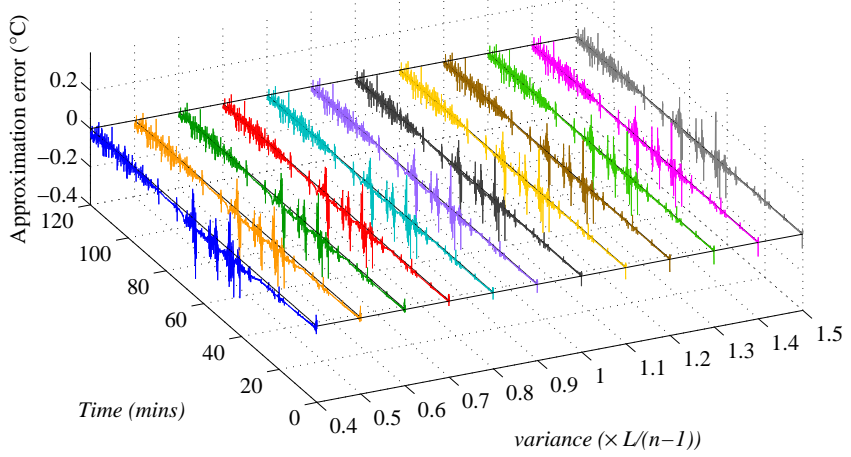

(e) $\mathrm{x}=\mathrm{L}$

Figure 11: Impact of the variance on the approximation error 
cluded that wider radial basis functions are favorable to achieve satisfactory results.

\subsection{Test4: Effect of the numerical grid size}

In this last series of numerical tests, we attend to evaluate the effect of the number of knots on the accuracy of the numerical approximate solutions. For this purpose, the approximation of the temperature evolution has been generated by changing the grid size in both numerical schemes, the semi discretization and the radial basis interpolation based scheme, considering (8) gaussian functions characterized by a variance equal to $\left(\frac{L}{n-1}\right)$. The test was repeated by gradually increasing the grid size (from 100 to 1100). Without loss of generality, the presented results display the density function of the computational errors introduced in both numerical schemes for two points on the collector tube $(L / 2$ and $L)$.

The chosen spatial resolution of the computational grid, i.e. the number of positions along the collector, represents a trade off between the desired approximation accuracy and the required computational effort. Control design studies would typically require a minimum number of state variables describing the system dynamics with an acceptable accuracy. Moreover, it is important to have an accurate numerical approximation of the transient response characterizing the real system behavior. Therefore, a high number of points might be required to achieve the desired performance under varying working conditions. The obtained results show that the higher is the number of the knots the higher is also the accuracy of the approximation of the numerical schemes (see Fig. 12). Indeed, the approximation errors of the gaussian interpolation and the semi discretization scheme are condensed in the neighborhood of the zero as the grid size $(p)$ increases. However, the state vector in the reduced order bilinear model is independent from the computational grid. Thereafter, the accuracy of the approximation can be improved by increasing the grid size without making the control design more complicated. Besides, for small grid sizes particularly for $(\mathrm{p}=100)$, the radial basis interpolation presents better performance. A too low number of discrete sections leads to an error varying between ( 0 and 10) at the position $\left(x=\frac{L}{2}\right)$ and between $(0$ and 20$)$ at the position $(x=L)$ comparing to an approximation error bounded by $( \pm 0.1)$ for the bilinear model (see Fig.13). Furthermore, the stability of the semi discrete technique is conditioned by the Courant-Friedrichs-Lewy (CFL) condition introducing an upper bound on the number of points used for the numerical approximation. This limitation in the spatial resolution can be illustrated by the results obtained for a grid of (1100) knots in Fig. 14. Indeed, the approximation error caused by the gaussian interpolation lies in the interval $([-0.04,0.02])$ while it is divergent for the semi discrete approximate model.

\section{Conclusion}

This work presents a new reduced order bilinear approximate model for the parabolic distributed solar collector. The proposed approximation is derived from the physical distributed

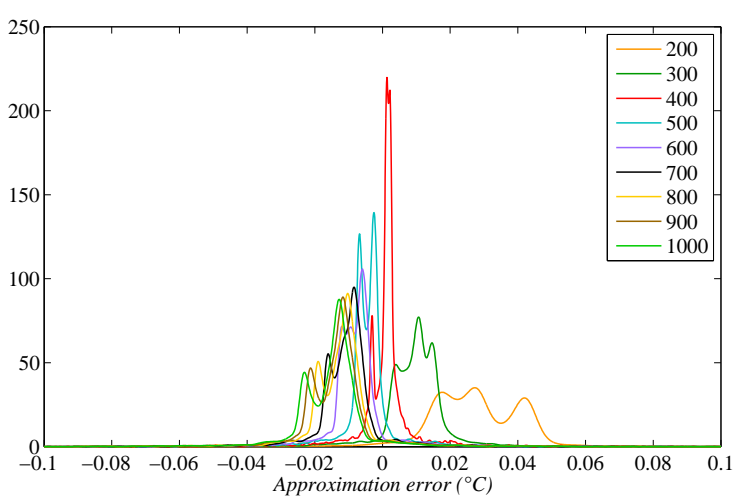

(a) Semi discrete model at $\mathrm{x}=\mathrm{L} / 2$

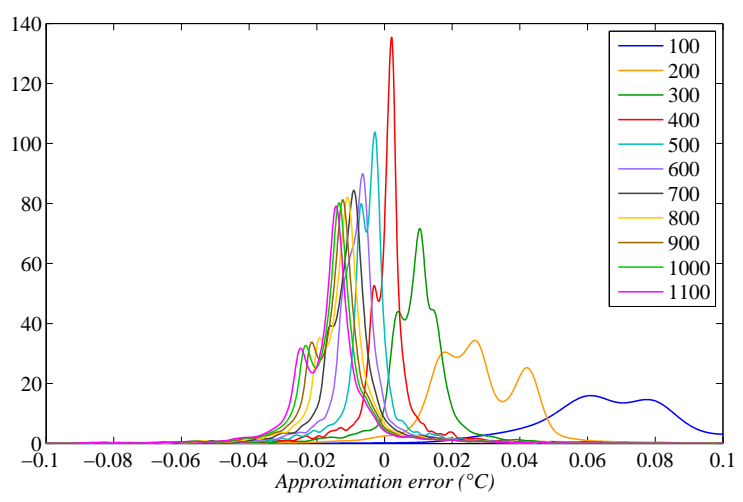

(b) Reduced bilinear model at $\mathrm{x}=\mathrm{L} / 2$

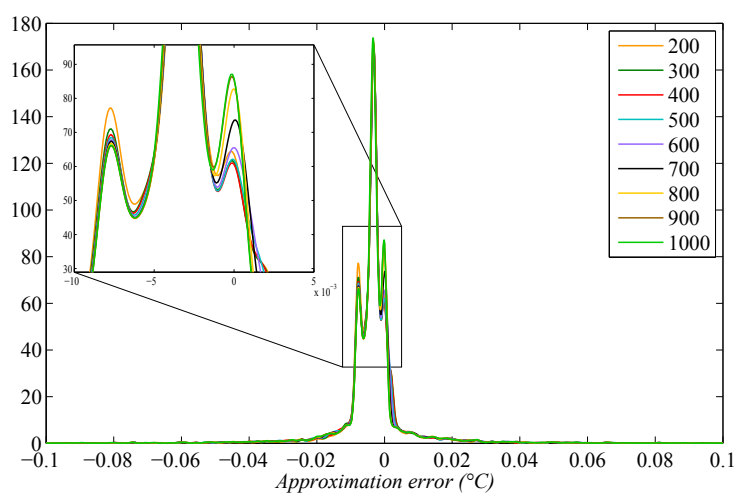

(c) Semi discrete model at $\mathrm{x}=\mathrm{L}$

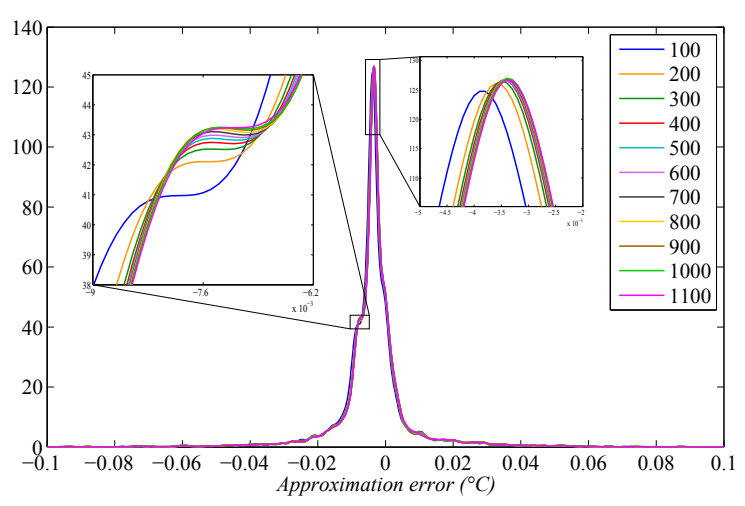

(d) Reduced bilinear model at $\mathrm{x}=\mathrm{L}$

Figure 12: Density distributions of the error engendered for different grid sizes 


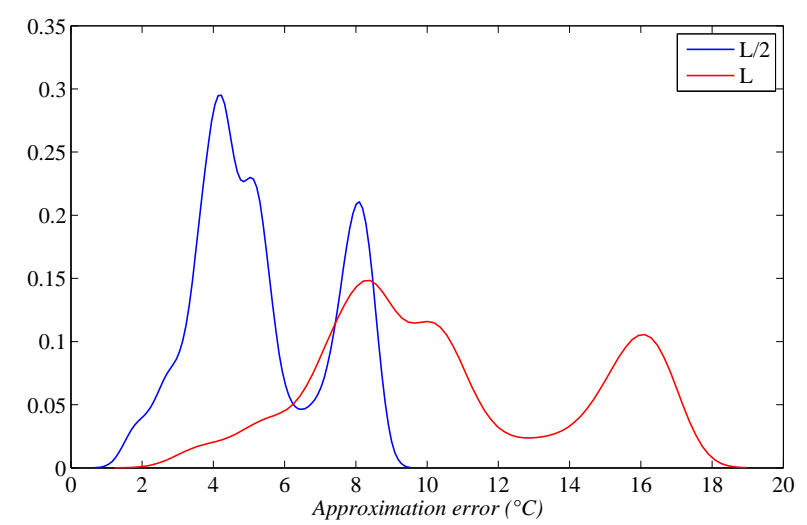

Figure 13: Density distribution of the error for $n=100$ using the semidiscretization

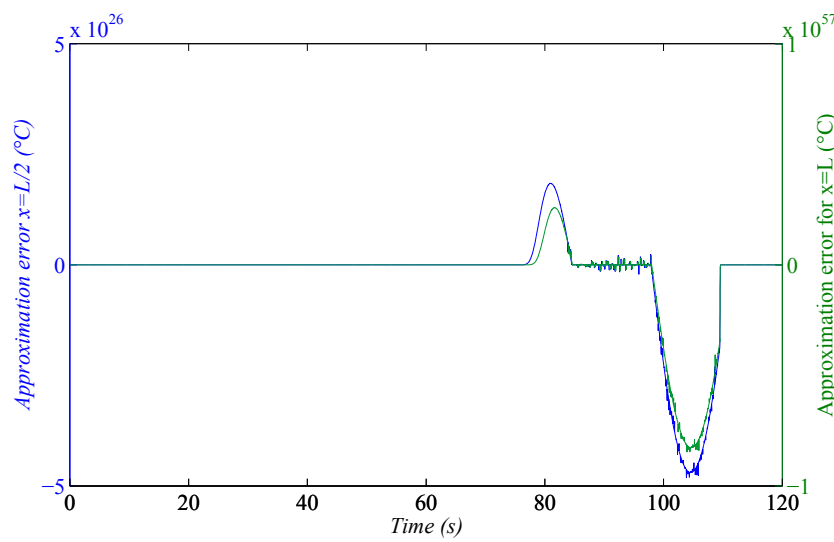

Figure 14: Evolution of the approximation error $n=1100$ using the semidiscretization

model describing the heat transport dynamics along the collector using a modified gaussian interpolation. The introduced lumped parameter representation is defined as a low dimensional state space model. The latter presents satisfactory results in terms of approximation accuracy. Moreover, it is characterized by its low dimension, which reduces the computational cost and simplifies the control design. In addition, the state dimension is independent from the size of the computational grid. Thereafter, the approximation accuracy might be improved without affecting or increasing the complexity of the controller. Hence, the proposed bilinear model presents promising features for model analysis and control design. The sensitivity of the approximation has been studied with respect to different parameters of the numerical scheme. Indeed, a gaussian interpolation based on $(n=8)$ sets considering a grid of $(p=500)$ along a collector tube, which has a total length of $(172 m)$, is able to give an accurate bilinear reduced mode for simulation purposes even under unfavorable conditions. These results are based on the assumption of a time varying source term considering the mirrors characteristics homogeneous in space with an equally distributed solar irradiance. The partial shading of the solar collector or the spatial inhomogeneity in the collector parameters can be easily taken into account in the bilinear reduced model without added complexity to the approximation procedure. It can be achieved by considering a spatio-temporal distributed source term $s(x, t)$ which affects consequently the matrix $B(t)$.

\section{Acknowledgment}

Research reported in this publication has been supported by King Abdullah University of Science and Technology (KAUST).

\section{References}

[1] L. Xu, Z. Wang, X. Li, G. Yuan, F. Sun, D. Lei, Dynamic test model for the transient thermal performance of parabolic trough solar collectors, Solar Energy 95 (2013) 65-78.

[2] E. F. Camacho, M. Berenguel, F. R. Rubio, Adanced control of solar plants, Springer, 1997.

[3] E. F. Camacho, F. R. Rubio, M. Berenguel, L. Valenzuela, A survey on control schemes for distributed solar collector fields. part i: Modeling and basic control approaches, Solar Energy 81 (2007) 1240-1251.

[4] Pickhardt, Nonlinear modeling and adaptive predictive control of a solar plant, Control Engineering Practice 8 (2000) 937-947.

[5] R. N. Silva, J. M. Lemos, L. M. Rato, Variable sampling adaptive control of a distributed collector solar field, IEEE Transactions on Control Systems Technology 11 (5) (2003) 813-823.

[6] G. Pin, M. Falchetta, G. Fenu, Adaptive time-warped control control of molten salt distributed collector solar fields, Control Engineering Practice 16 (2008) 813-823.

[7] A. J. Gallego, E. F. Camacho, Adaptive state space model predictive control of a parabolic trough field, Control Engineering Practice 20 (2012) 904-911.

[8] J. M. Igreja, J. M. Lemos, R. N. Silva, Adaptive receding horizon control of a distributed collector solar field, 44th IEEE Conference on Decision and Control, and the European Control Conference, Seville, Spain (Dec 2005).

[9] R. N. Silva, L. M. Rato, J. M. Lemos, Time scaling internal state predictive control of a solar plant, Control Engineering Practice 11 (2003) 1459-1467.

[10] E. J. Kansa, Multiquarics-a scattered data approximation scheme with applications to computational fluid-dynamics, Computers Math. Applic. $19(8 / 9)(1990)$ 147-161. 